Harvard Kennedy School Misinformation Review ${ }^{1}$

February 2022, Volume 3, Issue 1

Creative Commons Attribution 4.0 International (CC BY 4.0)

Reprints and permissions: misinforeview@hks.harvard.edu

DOI: https://doi.org/10.37016/mr-2020-92

Website: misinforeview.hks.harvard.edu

\title{
Leveraging infodemiologists to counteract online misinformation: Experience with COVID-19 vaccines
}

In the new information environment represented by the internet and social media platforms, information of public health importance is transmitted rapidly by decentralized, interpersonal networks rather than through traditional sources like public health officials or professional journalists, thus requiring a new approach to counteracting misinformation. We have previously advanced the idea that infodemiology, when combined with effective surveillance and diagnostics, can be an effective method for rapidly addressing online misinformation about science and health. Based on our experience with an infodemiology program aimed at misinformation about COVID-19 vaccinations, we can now offer general recommendations for more widespread training and deployment of infodemiologists who can rapidly respond in situations of high scientific uncertainty.

Authors: Jack M. Gorman (1), David A. Scales $(1,2)$

Affiliations: (1) Critica, USA, (2) Weill Cornell Medicine, USA

How to cite: Gorman, J. M., \& Scales, D. A. (2022). Leveraging infodemiologists to counteract online misinformation:

Experience with COVID-19 vaccines. Harvard Kennedy School (HKS) Misinformation Review, 3(1).

Received: December $9^{\text {th }}, 2021$. Accepted: February $3^{\text {rd }}, 2022$. Published: February $16^{\text {th }}, 2022$.

\section{Introduction}

To protect public health, officials must effectively communicate information of health relevance to the public. Traditionally, public health institutions carefully craft messages and issue them through websites, press conferences, and via community partners (Savoia et al., 2017). Such communications have been regarded by public health agencies as sufficient to meet their responsibilities for keeping the public informed.

The COVID-19 pandemic has, of course, shown us that this approach is insufficient because it prioritizes top-down communication, from experts to audience. In today's information environment, the "audience" has ceased to be entirely the receptive entity it once was; on internet and social media platforms people are simultaneously information consumers and producers (Kümpel, 2021), receiving information and reissuing it, often in dramatically altered form. One-way communication fails to take into account this new information environment in which every new scientific development can be commented upon even before public health officials have a chance to craft and issue their communications. Thus, there is a clear need to add a new form of public health communication that takes into account both the uncertainty of evolving science and the probability that any important new piece of information will be

\footnotetext{
${ }^{1}$ A publication of the Shorenstein Center on Media, Politics and Public Policy at Harvard University, John F. Kennedy School of Government.
} 
the subject of considerable online activity before experts can weigh in. We have previously advanced the idea that infodemiologists can be an effective method for rapidly addressing online misinformation about science and health when linked to effective infodemic surveillance and diagnostics (Scales et al., 2021).

\section{Why do we need infodemiologists?}

A case in point was the announcement last October that the U.S. Food and Drug Administration (FDA) authorized the use of a COVID-19 vaccine for children aged $5-12$ years old. This was a significant step in the effort to control the coronavirus epidemic, but it also generated immediate and substantial pushback from an array of vaccine skeptics. Consequently, many parents have expressed fears and reservations (Gerber \& Offit, 2021) about having their children vaccinated against COVID-19 and rates of vaccination in this age group may be lower than hoped for (Hamel et al., 2021). Explanations by public health officials and vaccine experts were reactive to the internet chatter that called into question the necessity, safety, and efficacy of vaccinating children against COVID-19.

This situation, which remains a significant public health setback, should have been anticipated, particularly in light of pre-existing hesitancy for childhood vaccines. Given what we know about circulating misinformation and disinformation about COVID-19 vaccines, it was predictable that misstatements and misconceptions about vaccinating children would emerge prior to and then increase immediately upon authorization. Public health communication responses could have been better prepared.

As part of the new paradigm for public health communication, we have recommended the creation of a corps of trained online interveners called infodemiologists, a term that was first introduced by Eysenbach (2002). Eysenbach defined infodemiology, or information epidemiology, as identifying "areas where there is a knowledge translation gap between best evidence...and practice..." We expand on Eysenbach's definition by incorporating a response protocol to bridge that gap, akin to how field epidemiology combines both disease tracking and epidemic response (Goodman et al., 2019). Here we explain in more detail our recommendations for infodemiology based on our experience with training and deploying infodemiologists to counteract online misinformation about COVID-19 vaccines.

\section{Who are infodemiologists?}

We envisage the hubs of the infodemiologist network to be composed of part-time people housed in different organizations like within government agencies like the U.S. Centers for Disease Control and Prevention (CDC); professional health societies, like the American Academy of Pediatrics (AAP); and nongovernmental organizations involved in healthcare improvement, like Critica. ${ }^{2}$ These public health institutions should all be ready to train infodemiologists and maintain a communication network between them. Actual infodemiologists can and should be people connected to various communities and networks and include community health workers, nurses, physicians, pharmacists, and community and faith leaders. These community liaisons should be trained in infodemiology but not necessarily using it all the time. Like people trained in cardiopulmonary resuscitation (CPR), they would have these skills at the ready, only to be deployed in the event of expected or current misinformation circulating in a community or network.

\footnotetext{
${ }^{2}$ Critica is a non-profit organization based in the Bronx, New York that seeks to improve the public's acceptance of scientific misinformation, counteract misinformation about health and science, and increase the use of scientific evidence in public policymaking.
} 
We have found that people with college and master's level degrees in a wide variety of disciplines are ideal infodemiologists, but even without those degrees, people with innate skills in communicating science to specific communities can make excellent infodemiologists. The important talent is the ability to be empathic and to communicate complicated scientific concepts in non-technical ways. Engagement is key to infodemiology.

To that end, the actual training of an infodemiologist is centered around methods for engagement and empathic understanding. Because studies have shown that science denial is usually not purely a knowledge deficit problem (Gorman \& Gorman, 2016; Kahan et al., 2011; McLintic, 2019) and that mere recitation of facts is often not sufficient to change attitudes and behaviors about health (Mclntyre, 2021) we have developed a protocol for intervention that is derived from several evidence-based techniques. We base our protocol around motivational interviewing (MI), a psychological approach to effecting change in behavior that is based on open-ended questions, establishing common ground, dealing with ambivalence, affirmation, and self-efficacy. It has been shown to be effective in a variety of settings, including substance use (Smedslund et al., 2011), medication adherence (Palacio et al., 2016), vaccine acceptance (Gagneur et al., 2019; 2018), and sexual risk-taking behavior (Starks et al., 2020). We combine an MI-based approach with reflective listening, providing accurate facts, and actively debunking disinformation. In the online setting, the intervention must be delivered in relatively short segments, one of the skills that infodemiologists must master. Our training consists of didactic modules and practice interventions with supervision and is delivered part-time over eight weeks, but a more condensed training could be done much more quickly.

Infodemiologists do not need to be scientific experts themselves, but it is imperative that infodemiologists be connected to relevant experts to ensure accuracy. In the example of COVID-19 vaccines, vaccine and infectious disease experts at academic institutions should be ready on short notice to answer questions posed by infodemiologists as they interact on internet and social media platforms. At the same time, infodemiologists need to be connected to each other to share best practices and aspects of on-the-ground surveillance.

\section{Infodemiology surveillance}

To be most effective, the corps of infodemiologists should also be connected to effective surveillance of misinformation, sometimes called "social listening" (Heldman et al., 2013). Given the breadth of reporting and commenting on health and science topics, no single entity or organization can possibly be responsible for the entire scope of misinformation surveillance. Therefore, we recommend that specialty societies, health-related NGOs, and government-based public health authorities share this task, each assuming responsibility for surveillance of specific areas. In doing so, these organizations should begin by identifying areas that have traditionally been most prone to misinformation, like vaccines and anti-cancer treatments (Johnson et al., 2021), while at the same time using their expertise to anticipate new areas of controversy that will likely stimulate misinformation.

Despite surveillance efforts, we expect infodemiologists will need to leverage a mixture of anticipating and reacting to misinformation. While the former is, of course, preferable in that it gives us more time to design educational outreach and the opportunity to prevent undesirable behaviors, like refusing vaccinations, there will inevitably be many times in which it is simply impossible to anticipate misinformation. The misinformation about COVID-19 vaccine efficacy that surrounded the death of Colin Powell was an example of the need to respond rapidly to a situation already in progress (Reuters Fact Check, 2021). The Powell situation exemplifies the critical need for an ongoing surveillance system to detect misinformation on the internet and to have infodemiologists who already have the trust of the 
communities and networks in which misinformation is spreading prepared to intervene rapidly, ideally within hours after it first appears on the internet.

\section{Where do infodemiologists intervene?}

While it is important to keep traditional media sources informed about the most up-to-date science regarding a health topic that is the subject of misinformation, it is equally important to recognize that most Americans have obtained at least some of their information about health from the internet for nearly a decade (Pew Research Center, 2013). This means that misinformation can spread throughout social media and affect behaviors long before scientists and healthcare experts issue their press releases. Even when these press releases do reach the media, it is uncertain how journalists and editors will use them. There is no guarantee that even the most carefully crafted press release will translate into a headline and story that truly sets the record straight on any given health topic.

For that reason, it is increasingly imperative for public health officials to partner with infodemiologytrained community liaisons to ensure high-quality public health information reaches the online networks where people actually obtain their health information in real-time. That is the critical place where these infodemiology-trained community liaisons must engage with misinformation, what we call "interventions," to ensure public health communication reaches throughout the decentralized networks of the internet and social media. Some of our observations learned through our infodemiologists' experience with interventions so far include:

1. Online interventions are best made as quickly as possible and not more than a few hours after misinformation has been posted. Through our infodemiologists' experience, we have found engaging while a conversation thread is fresh is more likely to lead to discussion.

2. Infodemiologists should make an initial assessment of the misinformation poster's "readiness for change" along the six stages of the transtheoretical model of behavioral change (Prochaska \& Velicer, 1997). This helps to stratify the type of hesitancy occurring and enables responding commensurately to where the poster is on that continuum (Gagneur et al., 2019). This segmentation approach is akin to Surgo Ventures' (2020) stratification of vaccine-related behavior into categories, from enthusiastic to receive the vaccine to skeptics unlikely to vaccinate under any circumstances, aiming to identify and assuage the concerns of the "persuadable middle."

3. Many people observe conversations taking place on social media without posting comments of their own. These "bystanders" may be seeking information and can potentially be misled by incorrect information. We train infodemiologists to keep bystanders in mind as they proceed with their interventions (Pederson et al., 2020), remembering that like the people who post misinformation, bystanders also exhibit a continuum of vaccine beliefs.

4. Infodemiologists' interventions need to be factually correct but not overly technical, a skill that infodemiologists hone only with practice. Stories and narratives can be more memorable than data (Dahlstrom, 2014), and infodemiologists are encouraged to use personal anecdotes when appropriate.

5. Infodemiologists who are connected to the community of interest and share their values are essential. For in situations of scientific uncertainty, values guide us, and an infodemiologist who shares the values of the target audience can not only provide factual information where it exists 
but help guide the audience through the uncertainty according to the shared values of the group. In our cases, intervening in Black and Latinx communities, this often takes the form of advocating for obtaining vaccines as a powerful way to empower communities and address a long history of medical racism and as a community-focused way to show one's love for their neighbor and their community (Carson et al., 2021; Dada et al., 2022).

\section{Conclusion}

We have outlined the basic elements of the infodemiology model, including why infodemiologists are needed, who they are, when they should intervene, and where and how they should intervene. It is, in our opinion, eminently feasible to recruit, train, and deploy a corps of part-time infodemiologists to meet the challenges to public health presented by the new elements of the health information environment. To continue only with one-way communication in traditional media and on static websites will be to miss the opportunity to effectively address the rapid flow of misinformation that threatens the public's health.

\section{Bibliography}

Carson, S. L., Casillas, A., Castellon-Lopez, Y., Mansfield, L. N., Morris, D., Barron, J., Ntekume, E., Landovitz, R., Vassar, S. D., Norris, K. C., Dubinett, S. M., Garrison, N. A., \& Brown, A. F. (2021). COVID-19 vaccine decision-making factors in racial and ethnic minority communities in Los Angeles, California. JAMA Network Open, 4(9), e2127582. https://doi.org/10.1001/jamanetworkopen.2021.27582

Dada, D., Djiometio, J. N., McFadden, S. M., Demeke, J., Vlahov, D., Wilton, L., Wang, M., \& Nelson, L. E. (2022). Strategies that promote equity in COVID-19 vaccine uptake for Black communities: A review. Journal of Urban Health. https://doi.org/10.1007/s11524-021-00594-3

Eysenbach, G. (2002). Infodemiology: The epidemiology of (mis)information. American Journal of Medicine, 113(9), 763-765. https://doi.org/10.1016/s0002-9343(02)01473-0

Gagneur, A., Battista, M.-C., Boucher, F. D., Tapiero, B., Quach, C., De Wals, P., Lemaitre, T., Farrands, A., Boulianne, N., Sauvageau, C., Ouakki, M., Gosselin, V., Petit, G., Jacques, M.-C., \& Dubé, È. (2019). Promoting vaccination in maternity wards - Motivational interview technique reduces hesitancy and enhances intention to vaccinate, results from a multicentre non-controlled preand post-intervention RCT-nested study, Quebec, March 2014 to February 2015. Euro Surveillance, 24(36). https://doi.org/10.2807/1560-7917.ES.2019.24.36.1800641

Gagneur, A., Gosselin, V., \& Dubé, È. (2018). Motivational interviewing: A promising tool to address vaccine hesitancy. Vaccine, 36(44), 6553-6555. https://doi.org/10.1016/j.vaccine.2017.10.049

Gerber, J. S., \& Offit, P. A. (2021). COVID-19 vaccines for children. Science, 374(6570). https://doi.org/10.1126/science.abn2566

Goodman, R. A., Buehler, J. W., \& Mott, J. A. (2019). Defining field epidemiology. The CDC Field Epidemiology Manual. https://www.cdc.gov/eis/field-epi-manual/chapters/Defining-FieldEpi.html

Gorman, S. E., \& Gorman, J. M. (2016). Denying to the grave: Why we ignore the facts that will save us. Oxford University Press.

Hamel, L., Lopes, L., Sparks, G., Kirzinger, A., Kearney, A., Stokes, M., \& Brodie, M. (2021, October 28). KFF COVID-19 vaccine monitor: October 2021. https://www.kff.org/coronavirus-covid-19/pollfinding/kff-covid-19-vaccine-monitor-october-2021/ 
Heldman, A. B., Schindelar, J., \& Weaver, J. B. (2013). Social media engagement and public health communication: Implications for public health organizations being truly "social." Public Health Reviews, 35(13). https://doi.org/10.1007/BF03391698

Johnson, S. B., Parsons, M., Dorff, T., Moran, M. S., Ward, J. H., Cohen, S. A., Akerley, W., Bauman, J., Hubbard, J., Spratt, D. E., Bylund, C. L., Swire-Thompson, B., Onega, T., Scherer, L. D., Tward, J., \& Fagerlin, A. (2021). Cancer misinformation and harmful information on Facebook and other social media: A brief report. JNCI: Journal of the National Cancer Institute, djab141. https://doi.org/10.1093/inci/djab141

Kahan, D. M., Jenkins-Smith, H., \& Braman, D. (2011). Cultural cognition of scientific consensus. Journal of Risk Research, 14(2), 147-174. https://doi.org/10.1080/13669877.2010.511246

Kümpel, A. S. (2021). Social media information environments and their implications for the uses and effects of news: The PINGS framework. Communication Theory, qtab012. https://doi.org/10.1093/ct/qtab012

Mclntyre, L. (2021). Talking to science deniers and sceptics is not hopeless. Nature, 596(7871), 165. https://doi.org/10.1038/d41586-021-02152-y

McLintic, A. (2019). The motivations behind science denial. The New Zealand Medical Journal, 132(1504), 88-94. https://journal.nzma.org.nz/journal-articles/the-motivations-behind-sciencedenial

Palacio, A., Garay, D., Langer, B., Taylor, J., Wood, B. A., \& Tamariz, L. (2016). Motivational interviewing improves medication adherence: A systematic review and meta-analysis. Journal of General Internal Medicine, 31(8), 929-940. https://doi.org/10.1007/s11606-016-3685-3

Pew Research Center. (2013, February 1). Majority of adults look online for health information. https://www.pewresearch.org/fact-tank/2013/02/01/majority-of-adults-look-online-for-healthinformation/

Prochaska, J. O., \& Velicer, W. F. (1997). The transtheoretical model of health behavior change. American Journal of Health Promotion, 12(1), 38-48. https://doi.org/10.4278\%2F0890-1171-12.1.38

Reuters Fact Check. (2021). Fact check - Death of Colin Powell is not evidence that vaccines are ineffective; medical history put him at great risk. https://www.reuters.com/article/factcheckcolin-powell-covid19/fact-check-death-of-colin-powell-is-not-evidence-that-covid-19-vaccinesare-ineffective-medical-history-put-him-at-greater-risk-idUSL1N2RE2LY

Savoia, E., Lin, L., \& Gamhewage, G. M. (2017). A conceptual framework for the evaluation of emergency risk communications. American Journal of Public Health, 107(S2), S208-S214. https://doi.org/10.2105/AJPH.2017.304040

Scales, D., Gorman, J., \& Jamieson, K. H. (2021). The COVID-19 infodemic - Applying the epidemiologic model to counter misinformation. New England Journal of Medicine, 385(8), 678-681. https://doi.org/10.1056/NEJMp2103798

Smedslund, G., Berg, R. C., Hammerstrøm, K. T., Steiro, A., Leiknes, K. A., Dahl, H. M., \& Karlsen, K. (2011). Motivational interviewing for substance abuse. Campbell Systematic Reviews, 7(1), 1-27. https://doi.org/10.4073/csr.2011.6

Starks, T. J., Robles, G., Doyle, K. M., Pawson, M., Bertone, P., Millar, B. M., \& Ingersoll, K. S. (2020). Motivational interviewing with male couples to reduce substance use and HIV risk: Manifestations of partner discord and strategies for facilitating dyadic functioning. Psychotherapy, 57(1), 58-67. https://doi.org/10.1037/pst0000278

Surgo Ventures. (2020). Vaccine persona explainer. https://surgoventures.org/vaccine-personaexplainer 


\section{Acknowledgements}

The authors wish to thank Dr. Oktawia Wojcik and Ms. Nancy Barrand for their help in pursuing this research and the Critica infodemiologists for conducting many of the online interventions upon which this paper is based.

\section{Funding}

Support for this article was provided in part by the Robert Wood Johnson Foundation (\#76935) and Weill Cornell Medicine's JumpStart Research Career Development Program. The views expressed here do not necessarily reflect those of the Foundation or Weill Cornell.

\section{Competing interests}

None.

\section{Copyright}

This is an open access article distributed under the terms of the Creative Commons Attribution License, which permits unrestricted use, distribution, and reproduction in any medium, provided that the original author and source are properly credited. 